\title{
Investigating variations of the electron beam voltage on the traveling wave tube output power in the different frequencies
}

\author{
F. Sheykhe ${ }^{1} \cdot$ Sh. Saviz ${ }^{1} \cdot$ M. Salem ${ }^{1}$
}

Received: 14 May 2018 / Accepted: 17 July 2018 / Published online: 25 July 2018

(C) The Author(s) 2018

\begin{abstract}
In the present research, the effect of variations of the electron beam voltage on the output power is studied. In order to develop the study, this problem was investigated at different frequencies, which are the products of nonlinear behavior of the traveling wave tube (TWT) in response to the input frequency. Moreover, for a more realistic understanding, the tubes were considered with two linear and nonlinear responses to the input frequency. The TWT output power was calculated in linear and nonlinear modes, at different frequencies using the numerical solution of the mathematical equations of the Lagrangian model. Then, the output power in terms of distance and beam voltage in different frequencies was plotted and compared. The results revealed that the effects of variations of voltage on the output power were more proper in a singlemode TWT in comparison with a multimode one.
\end{abstract}

Keywords Beam voltage $\cdot$ Output power $\cdot$ Nonlinear $\cdot$ Traveling wave tube

\section{Introduction}

Traveling wave tubes (TWTs) are devices that are widely used in communication, electronic warfare and radar systems $[1,2]$. These devices have wide bandwidths, highfrequency and high-power operating points; as a result, they have wider spread application. The nonlinear behaviors of the TWTs are known as one of the important practical limits of them. Until now, many efforts have been made to reduce these nonlinear effects $[7-9,11,12]$. The nonlinearity features are recognized as spectral distortions and saturating mechanism [3]; both of which decrease the efficiency of the TWT.

For single-tone mode, nonlinear distortion products appear as harmonics products $(f, 2 f, 3 f \ldots)$, while for multi tone mode (multicarrier operation) nonlinear distortion products appear as intermodulation products $(\mathrm{mf} 1+\mathrm{nf} 2)$ at the output of the amplifier [3]. In this work, the effects of changes in beam voltage on the output power of TWTs are

Sh. Saviz

shahrooz.saviz@srbiau.ac.ir; azarabadegan@gmail.com

1 Plasma Physics Research Center, Science and Research Branch, Islamic Azad University, Tehran, Iran studied in both the presence and absence of a nonlinear phenomenon (spectral distortion).

The most important mechanism that occurs inside the tube is the interaction between the input wave and the electron beam. When an electron beam is injected along the axis of the helix, electric field horizontal component of the wave accelerates some electrons and decelerates the others. This is the basis for the formation of bunch of the electron beam and the transfer of energy from the beam to the wave and ultimately the amplification of the output wave $[1,2]$. Electron beam emission voltage into the tube is the source of energy and beam velocity. Therefore, determination of the cathode voltage and its variations, which are the emitter of the electrons from the gun and called the beam voltage, is very important. In many previous attempts, the basis for selecting the voltage for the beam was the TWT efficiency and maximum output power. In the previous studies, the value of the beam voltage or the cathode voltage was equal to $3150 \mathrm{~V}[6,10,15]$. In other studies, this value was considered as $2750 \mathrm{v}$ in [11], $4350 \mathrm{v}$ in [5], $4880 \mathrm{v}$ in [13] and $4920 \mathrm{v}$ in [14]. Therefore, in this study, the effects of voltage changes on the improvement in TWT output power is studied using numerical solution of the governing equations. The TWT is modeled by several authors using the Eulerian and Lagrangian electron beam coordinates [4]. 
Among all of the present models, the multi-frequency spectral Eulerian (MUSE) and Lagrangian TWT equations (LATTE) are the most important physical models. The basis of using these two models is the physical interpretation used for the electron beam as a fluid [4]. In "Formulation" section, we formulate the governing equations for the Lagrangian model of the TWT. The numerical solutions as well as the diagrams which show output power in terms of voltage and distance are presented in "Numerical results" section. Finally, the discussion and conclusion of the numerical results are given in "Discussion and Conclusion" section.

\section{Formulation}

The transmission line, Poisson, continuity and Vlasov equations are used to derive the mathematical equations of the TWT which are expressed in the domain of time as follows $[3,4,16]$ :

Transmission line equations:

$\frac{\partial V}{\partial z}=h_{1} \frac{\partial I}{\partial t}$

$\frac{\partial I}{\partial z}=h_{2} \frac{\partial V}{\partial t}-A \frac{\partial \rho}{\partial t}$

In Eqs. (1) and (2), the current and voltage are expressed with I and $\mathrm{V}$, respectively. The $\rho$, volume charge density, $t$, time and $z$ is the axial distance.

Poisson's equation:

$\frac{\partial E}{\partial z}=\frac{\rho}{\varepsilon_{0}}$

In Eq. 3, $E$ is the space charge electric field.

Continuity and Vlasov equations:

$\frac{\partial v}{\partial t}+v\left(\frac{\partial v}{\partial z}\right)=-\frac{e}{m_{e}} h_{1}\left(\frac{\partial I}{\partial t}\right)+\frac{e}{m_{e}} \Re E$

$\frac{\partial \rho}{\partial t}+v\left(\frac{\partial \rho}{\partial z}\right)=-\rho\left(\frac{\partial v}{\partial z}\right)$

In Eqs. (1) and (2), the coefficients of and are the Fourier transforms inverse that are expressed as follows:

$h_{1}(z, t)=F^{-1}\left\{\frac{\tilde{K}\left(z, f_{l} \omega_{0}\right)}{\tilde{v}_{\mathrm{ph}}\left(z, f_{l} \omega_{0}\right)}\right\}$

$h_{2}(z, t)=F^{-1}\left\{\frac{1}{\tilde{v}\left(z, f_{l} \omega_{0}\right) \tilde{K}\left(z, f_{l} \omega_{0}\right)}\right\}$

$\Re(z, t)=F^{-1}\left\{\Re\left(z, f_{l} \omega_{0}\right)\right\}$

where the functions $\tilde{v}_{\mathrm{ph}}\left(z, f_{l} \omega_{0}\right), \tilde{K}\left(z, f_{l} \omega_{0}\right)$ and $\Re\left(z, f_{l} \omega_{0}\right)$ are defined as cold circuit phase velocity, frequency domain circuit interaction impedance and space charge reduction factor, respectively.

Using the furrier transform, Eqs. (1), (2), (3), (4) and (5) are transformed from the time domain to the frequency domain.

Using the coordinate transformation in Eqs. (9) and (10), where $z$ is the distance and $\psi$ is the phase. The phase is described with respect to a traveling wave of speed $u_{0}$ and frequency $\omega_{0}$

$z=z$

$\psi=\omega_{0}\left(\frac{z}{u_{0}}-t\right)$

According to Fig. 1, in which, $R$ is the resistance, $C$ is capacitance, $G$ is shunt, and $L$ is the inductive series; applying Kirchhoff's voltage and current laws, Eqs. (1) and (2) are as follows.

$\frac{\partial V}{\partial z}=R I-\frac{\omega_{0}}{u_{0}} \frac{\partial V}{\partial \psi}-\omega_{0} L \frac{\partial I}{\partial \psi}$

$\frac{\partial I}{\partial z}=G V-\omega_{0} C \frac{\partial V}{\partial \psi}-\frac{\omega_{0}}{u_{0}} \frac{\partial I}{\partial \psi}+A \omega_{0} \frac{\partial \rho}{\partial \psi}$

If Eqs. (9) and (10) are used, Eqs. (3), (4) and (5) are derived as follows.

$\frac{\partial E}{\partial z}=-\frac{\omega_{0}}{u_{0}} \frac{\partial E}{\partial \psi}+\frac{\rho}{\varepsilon_{0}}$

$v \frac{\partial v}{\partial z}=\frac{e}{m_{e}}\left(\omega_{0} L \frac{\partial I}{\partial \psi}-R I\right)+\frac{e}{m_{e}} \Re E+\omega_{0}\left(1-\frac{v}{u_{0}}\right) \frac{\partial v}{\partial \psi}$

$v \frac{\partial \rho}{\partial z}=\omega_{0}\left(1-\frac{v}{u_{0}}\right) \frac{\partial \rho}{\partial \psi}-\rho\left(\frac{\partial v}{\partial z}+\frac{\omega_{0}}{u_{0}} \frac{\partial v}{\partial \psi}\right)$

In Eq. $14, v$ is the electron beam velocity and $\Re$ is space charge reduction factor.

Also, $m_{e}$ and $e$ are, respectively, the mass and the charge of the electron.

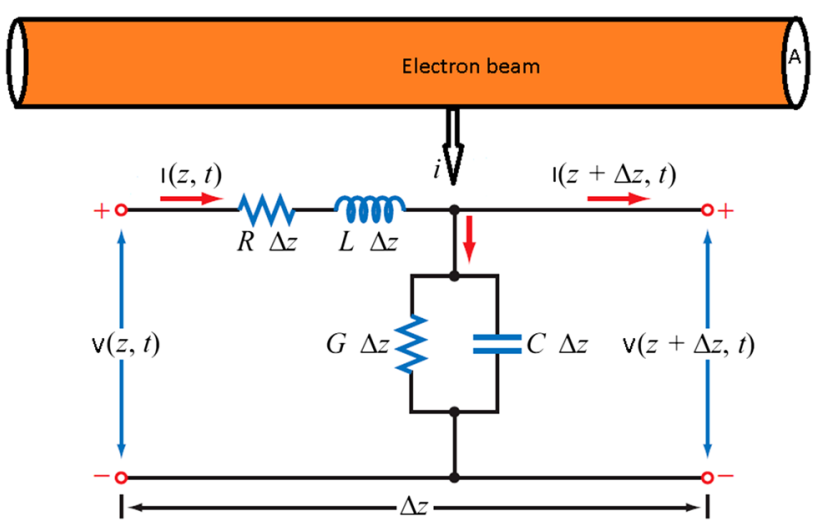

Fig. 1 Each section of the helix is represented by an equivalent circuit [16] 
According to the above discussion, each TWT contains three main parts, the slow wave structure, the source of the electron beam and the propagation of an electromagnetic wave that have approximately the same phase velocity with electron beam. The mathematical Eqs. (11)-(15) form a differential equation system which describes the mechanism of the TWT. In order to normalize the quantities used, the following characteristic quantities are defined:

$z=L$

$U=u_{0}$

$T=\frac{z}{U}$

In the above equations, $u_{0}$ is the DC beam velocity, $L$ is the TWT circuit length, and $T$ is a characteristic time. The following variables illustrate the normalization of dependent variables and independent coordinates $[3,4,16]$.

Independent coordinates:

$\hat{z}=\frac{z}{L}$

$\hat{\psi}=\frac{\psi}{\omega_{0} T}=(\hat{z}-\hat{t})$

$\hat{t}=\frac{t}{T}$.

Dependent variables:

$\tilde{V}=\frac{C}{K I_{0}} V$

$\tilde{I}=\frac{C}{I_{0}} I$

$\tilde{E}=\frac{\varepsilon_{0}}{L \rho_{0}} E$

$\tilde{\rho}=\frac{\rho}{\rho_{0}}$

$\tilde{v}=\frac{v}{u_{0}}$.

Normalized quantities:

$\hat{R}=R \frac{L}{K}$

$\hat{L}=L \frac{U}{K}$

$\hat{G}=G(Z K)$

$\hat{C}=(U K)$

$\hat{\Re}=\frac{e \rho_{0} T^{2}}{m_{e} \varepsilon_{0}} \Re=\omega_{p}^{2} T^{2} \Re$

Derivatives with respect to $z$ and then $\psi$ become $\frac{\partial}{\partial z}=\frac{1}{L} \frac{\partial}{\partial \hat{z}}$

$\frac{\partial}{\partial \psi}=\frac{1}{\omega_{0} T} \frac{\partial}{\partial \hat{\psi}}$

Some other relationships between DC parameters can be written as follows:

$\mathbb{C}=\left(\frac{K I_{0}}{4 V_{0}}\right)^{1 / 3}$

$I_{0}=\rho_{0} u_{0} A$

$u_{0}=\left(\frac{2 e V_{0}}{m_{e}}\right)^{1 / 2}$

In Eqs. (34)-(36), $\rho_{0}$ is the DC linear charge density, $V_{0}$ is the DC beam voltage, $I_{0}$ is the DC beam current, and $\mathbb{C}$ is the Pierce gain parameter.

The function $x(z, \psi)$ is the Fourier series relations that is defined as follows $[3,4,16]$

$x(z, \psi)=\sum_{j=-\infty}^{\infty} \tilde{x}_{j}(z) e^{i f_{j} \psi}$

$\tilde{x}_{j}(z)=\frac{1}{2 \pi} \int_{2 \pi} x(z, \psi) e^{-i f_{j} \psi} \mathrm{d} \psi$

where,

$f_{j}=\left(m f_{1} \pm n f_{2}\right)$

$f_{j}$ is the set of frequencies that are the drive frequencies together with the frequencies produced from nonlinear interactions, $\mathrm{m}$ and $\mathrm{n}$ are the integers positive and negative, and $\psi$ is the periodic function.

$(z, \psi)$ are Eulerian independent variables and $\left(z, \psi_{0}\right)$ are Lagrangian independent variables, where $z$ is axial position and $\psi$ is phase, and $\psi_{0}$ is the phase position of a fluid element with respect to the stream wave. The transformation from Lagrangian to Eulerian coordinates is given by functions $\mathrm{Z}$ and $\psi$

$\left[\begin{array}{l}z \\ \psi\end{array}\right]=\left[\begin{array}{l}Z\left(z, \psi_{0}\right) \\ \Psi\left(z, \psi_{0}\right)\end{array}\right]$

$Z\left(z, \psi_{0}\right)$ is the axial position of fluid element $\psi_{0}$ at $\mathrm{z}$ so $Z\left(z, \psi_{0}\right)=z$.

A function $g_{\mathrm{E}}$ of Eulerian variables $(z, \psi)$ is transformed to a function $g_{\mathrm{L}}$ of Lagrangian variables $\left(z, \psi_{0}\right)$ using

$g_{\mathrm{L}}\left(z, \psi_{0}\right)=g_{\mathrm{E}}\left(Z\left(z, \psi_{0}\right), \Psi\left(z, \psi_{0}\right)\right)$

According to Eqs. (40) and (41), transformation matrix of equation is as follows: 


$$
\left[\begin{array}{ll}
\frac{1}{\partial \Psi} & \frac{\partial}{\partial \psi} \\
\frac{\partial \psi_{0}}{\partial \psi_{0}}
\end{array}\right]
$$

That Jacobin matrix equals to:

$$
J=\frac{\partial \Psi}{\partial \psi_{0}}
$$

The partial derivatives are transformed as follows

$$
\left[\begin{array}{cc}
\frac{\partial}{\partial z} & \frac{\partial}{\partial \psi}
\end{array}\right]=\left[\begin{array}{ll}
\frac{\partial}{\partial z} & \frac{\partial}{\partial \psi_{0}}
\end{array}\right]\left[\begin{array}{ll}
1 & 0 \\
\frac{\partial \Psi}{\partial z} & \frac{\partial \Psi}{\partial \psi_{0}}
\end{array}\right]^{-1}
$$

According to a certain method in the Lagrangian model, we have:

$\frac{\partial \Psi}{\partial z}=\frac{\omega_{0}}{u_{0}}\left(1-\frac{u_{0}}{v_{L}}\right)$

Using Eq. (46), convective derivative in the Eulerian coordinates becomes

$v_{\mathrm{E}} \frac{\partial v_{\mathrm{E}}}{\partial z}+\omega_{0}\left(\frac{v_{\mathrm{E}}}{u_{0}}-1\right) \frac{\partial v_{\mathrm{E}}}{\partial \psi}=v_{\mathrm{L}} \frac{\partial v_{\mathrm{L}}}{\partial z}$

Applying derivative transformation relation (45) to the continuity Eq. (15), the following relation is obtained:

$\frac{\partial \Psi}{\partial \psi_{0}} \frac{\partial \rho_{\mathrm{L}} v_{\mathrm{L}}}{\partial z}=-\omega_{0} \frac{\partial \rho_{\mathrm{L}}}{\partial v_{\mathrm{L}}} \frac{\partial v_{\mathrm{L}}}{\partial \psi_{0}}$

Derivative from Eq. (46) relative to $\psi_{0}$ one gets

$\frac{\partial}{\partial z} \frac{\partial \Psi}{\partial \psi_{0}}=\frac{\omega_{0}}{\left(v_{L}\right)^{2}} \frac{\partial v_{L}}{\partial \psi_{0}}$

Substitute (49) into (48) and integrate to get

$\frac{\partial \Psi}{\partial \psi_{0}} \rho_{\mathrm{L}} v_{\mathrm{L}}=\kappa$

In which $\kappa$ is a constant of integration. By definition $\Psi\left(0, \psi_{0}\right)=\psi_{0}$, the values $\frac{\partial \Psi}{\partial \psi_{0}}$ and $\rho_{\mathrm{L}} v_{\mathrm{L}}$ on the $\psi_{0}$ axis, are $\frac{\partial \Psi\left(0, \psi_{0}\right)}{\partial \psi_{0}}=1$ and $\rho_{0} v_{0}$, respectively. Considering the following equation

$\rho_{0}\left(0, \psi_{0}\right) v_{0}\left(0, \psi_{0}\right)=\frac{I_{0}\left(0, \psi_{0}\right)}{A}$

Equation (50) becomes

$\left|\frac{\partial \Psi}{\partial \psi_{0}}\right| \rho_{L}\left(0, \psi_{0}\right) v_{L}\left(0, \psi_{0}\right)=\kappa=\frac{I_{0}\left(\psi_{0}\right)}{A}$

The Fourier coefficient of $\rho_{E}$ is expressed as

$\tilde{\rho}_{\mathrm{E}}^{l}=\frac{1}{2 \pi} \int_{2 \pi} \rho_{\mathrm{E}} \mathrm{e}^{-i f_{l} \psi} \mathrm{d} \psi$

Pulling Eq. (53) back to Lagrangian coordinates (for fixed $z$ ) and using Eq. (44) one gets

$$
\begin{aligned}
\tilde{\rho}_{\mathrm{E}}^{l} & =\frac{1}{2 \pi} \int_{2 \pi} \rho_{\mathrm{L}}\left|\frac{\partial \Psi}{\partial \psi_{0}}\right| \mathrm{e}^{-i f_{l} \Psi\left(0, \psi_{0}\right)} \mathrm{d} \psi_{0} \\
& =\frac{1}{2 \pi} \int \frac{I_{0}\left(0, \psi_{0}\right)}{A v_{L}\left(0, \psi_{0}\right)}\left|\frac{\partial \Psi}{\partial \psi_{0}}\right| \mathrm{e}^{-i f_{l} \Psi\left(0, \psi_{0}\right)} \mathrm{d} \psi_{0}
\end{aligned}
$$

Using Eqs. (37), (38) and (54), the circuit equations, space charge equation, Newton's law and phase relation (11-15), in Lagrangian coordinates, the following equations are created

$\frac{\mathrm{d} \tilde{V}_{j}}{\mathrm{~d} z}=-\frac{i f_{j} \omega_{0}}{u_{0}} \tilde{V}_{j}-\frac{i f_{j} \omega_{0} \tilde{K}\left(f_{j} \omega_{0}\right)}{\tilde{v}_{\mathrm{ph}}\left(f_{j} \omega_{0}\right)} \tilde{I}_{j}$

$$
\begin{aligned}
\frac{\mathrm{d} \tilde{I}_{j}}{\mathrm{~d} z}= & -\frac{i f_{j} \omega_{0}}{\tilde{K}\left(f_{j} \omega_{0}\right) \tilde{v}_{\mathrm{ph}}\left(f_{j} \omega_{0}\right)} \tilde{V}_{j}-\frac{i f_{j} \omega_{0}}{u_{0}} \tilde{I}_{j} \\
& +i f_{j} \omega_{0} \frac{1}{2 \pi} \int_{2 \pi} \frac{I_{0}\left(\psi_{0}\right)}{v\left(z, \psi_{0}\right)} \mathrm{e}^{-i f_{j} \Psi\left(z, \psi_{0}\right)} \mathrm{d} \psi_{0}
\end{aligned}
$$

$\frac{\mathrm{d} \tilde{E}_{j}}{\mathrm{~d} z}=-\frac{i f_{j} \omega_{0}}{u_{0}} \tilde{E}_{j}+\frac{1}{\varepsilon_{0} A} \frac{1}{2 \pi} \int_{2 \pi} \frac{I_{0}\left(\psi_{0}\right)}{v\left(z, \psi_{0}\right)} \mathrm{e}^{-i f_{j} \Psi\left(z, \psi_{0}\right)} \mathrm{d} \psi_{0}$

$\frac{\partial v}{\partial z}=\frac{1}{v} \sum_{-\infty}^{+\infty}\left\{\frac{i f_{j} \omega_{0} e \tilde{K}\left(f_{j} \omega_{0}\right)}{m_{e} \tilde{v}_{\mathrm{ph}}\left(f_{j} \omega_{0}\right)} \tilde{I}_{j}+\frac{e}{m_{e}} \Re\left(f_{j} \omega_{0}\right) \tilde{E}_{j}\right\} \mathrm{e}^{i f_{j} \Psi\left(z, \psi_{0}\right)}$

$\frac{\partial \Psi}{\partial z}=\frac{\omega_{0}}{u_{0}}\left(1-\frac{u_{0}}{v}\right)$

where $\quad-\infty<j<\infty, \quad$ and $\quad f_{j}=f_{m}+f_{n}, \quad$ for $m>n, f_{m}>f_{n}$.

For practical implementation, one neglects higher frequencies and limits to $-M<j<M$.

The equations in (55-59) are ordinary differential equations, and we will use standard ordinary differential equation integration techniques. The problem is an initial value problem, where one can calculate the proper initial values using (38). Otherwise for, $j \neq 0, \tilde{E}_{j}=\tilde{v}_{j}=\tilde{\rho}_{j}=0$, also for $\quad j=0, \tilde{E}_{0}=\tilde{V}_{0}=\tilde{I}_{0}=0 \quad$ and $\tilde{v}_{0}(0)=u_{0}, \tilde{\rho}_{0}(0)=\rho_{0}$.

In addition to the initial conditions mentioned above, there are other parameters that are very important in the performance of the TWT. These constant values are given in Table 1.

\section{Numerical results}

In this work, different voltages have been applied to the electron beam in the assumed frequency range in order to evaluate the TWT response. In this process among different mathematical models, the Lagrangian mathematical model 
Table 1 Constant values

\begin{tabular}{lllll}
\hline Parameter & Value & Parameter & Value & Parameter \\
\hline Electron mass $\left(m_{e}\right)$ & $9.11 \times 10^{-31} \mathrm{~kg}$ & Circuit length $(L)$ & $0.42 \mathrm{~m}$ & Number of circuit sections \\
Electron charge $(e)$ & $1.6 \times 10^{-19} \mathrm{c}$ & Beam outer radius & $0.0009652 \mathrm{~m}$ & Number dispersion frequency \\
Helix radius & $0.002353 \mathrm{~m}$ & Beam inner radius & 0.0 & \\
Helix wire width $(\mathrm{w})$ & $0.000305 \mathrm{~m}$ & Beam current $\left(I_{0}\right)$ & $0.0655 \mathrm{~A}$ & Base frequency $\left(\omega_{0}\right)$
\end{tabular}

has been applied on the TWT, which includes all the nonlinear features of a TWT properly. Accordingly, the governing equations on TWTs which indicate the changes in voltage and current circuit, space charge field as well as velocity and density of the electron beam are written. Also by applying the initial conditions appropriate to the TWT features and using fixed step fourth-order Runge-Kutta integrator numerical method, the differential equation systems 55-59 have been solved.

In this study, the TWT has been examined in two different situations; as a result, two different responses are reached which are discussed below:

\section{Situation (1)}

In this case, the response of the TWT is examined under the influence of applying the voltage in the absence of harmonic and intermodulation frequencies (single-tone mode). In this situation, the operation of the TWT was studied in the single frequency mode, which means the output frequency from the TWT is the same as the single input frequency entered to it. The final results of the calculations in this mode are revealed in diagrams $2-3$ which display output power in terms of distance (z), voltage (v) and frequency $(f)$.

In Fig. 2, the output power of the circuit is plotted at frequencies of 1400,1600 and $1800 \mathrm{MHz}$, in terms of different voltage of the electron beam. In this case, the circuit power is calculated for each single input frequency, the harmonic frequencies and the intermodulation are not

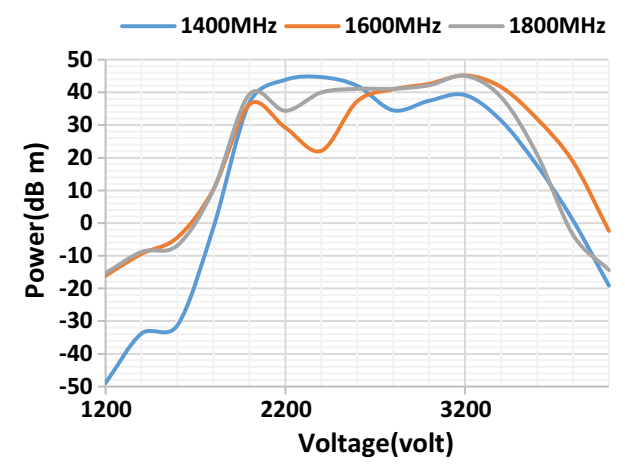

Fig. 2 Circuit output power versus of the electron beam voltages

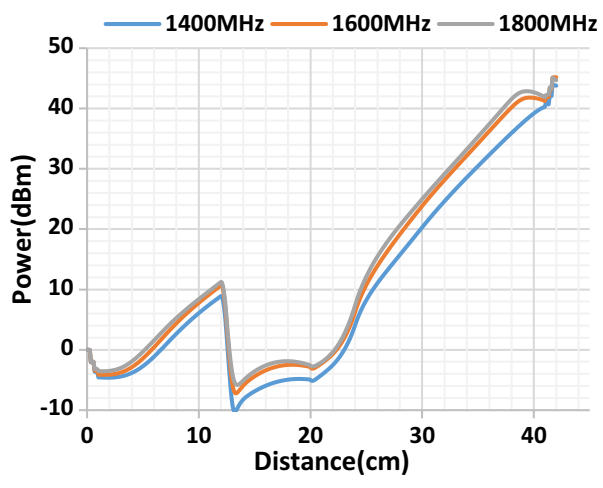

Fig. 3 Circuit output power versus of the axial distance, $V=3200 \mathrm{v}$

produced. In the voltage range of $2000-3300 \mathrm{v}$, the amplification of the wave at these three frequencies is approximately constant.

In Fig. 3, the output power is plotted in terms of axial distance, for a voltage of $3200 \mathrm{v}$ at frequencies of 1400 , 1600 and $1800 \mathrm{MHz}$. It is noticeable that the circuit power is saturated at $40 \mathrm{~cm}$ in all the three frequencies. According to Fig. 2, if the voltage of 2600 was selected, the amplification of all the three frequencies would occur alike.

\section{Situation (2)}

In this case, the response of the TWT is examined under the influence of applying the voltage in the presence of drive, harmonic and intermodulation frequencies (multi tone mode). Here, when two input frequencies which their difference is about $1 \mathrm{MHz}$ are entered to the TWT, drive frequencies as well as unwanted harmonic and intermodulation output frequencies are produced (Table 2). The final results of the calculations in this mode are revealed in diagrams 4-8 which display output power in terms of distance $(z)$, voltage $(v)$ and frequency $(f)$.

In Fig. 4, the output power of the circuit is plotted at frequencies of 1400,1600 and $1800 \mathrm{MHz}$, in terms of different voltage of the electron beam. In this case, the two main input frequencies are considered (Table 2); as a result, harmonic and intermodulation frequencies are produced. In the voltage range of $2000-2800 \mathrm{v}$, the difference in amplification of the wave at these three frequencies is small (Fig. 5). 
Table 2 Nonlinear second- and third-order products created for two input signals

\begin{tabular}{lllll}
\hline Input frequency $(\mathrm{MHz})$ & \multicolumn{4}{l}{ Frequencies generated by the TWT $(\mathrm{MHz})$} \\
\cline { 2 - 5 } Driven-order & First-order & Second-order harmonics & Third-order & \\
\cline { 3 - 5 } & & & Harmonics & Intermodulation \\
\hline 1400,1401 & 1400,1401 & $1,2800,2801,2802$ & 4200,4203 & $1399,1402,4201,4202$ \\
1600,1601 & 1600,1601 & $1,3200,3201,3202$ & 4800,4803 & $1599,1602,4801,4802$ \\
1800,1801 & 1800,1801 & $1,3600,3601,3602$ & 5400,5403 & $1799,1802,5401,5402$ \\
\hline
\end{tabular}

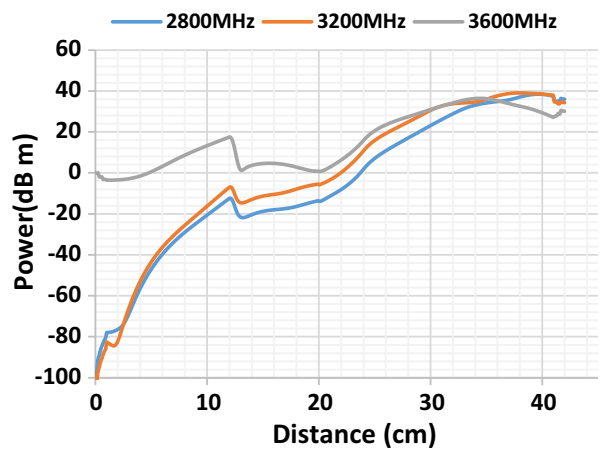

Fig. 6 Circuit output power versus axial distance, $V=3200 \mathrm{v}$

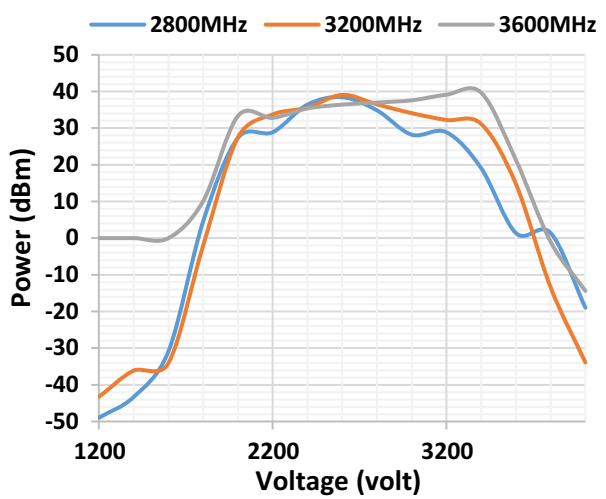

Fig. 7 Circuit output power versus beam voltage

$2000-2600 \mathrm{v}$, it is clear that the output power difference in the circuit is small in these three frequencies.

In Fig. 8, the output power of the circuit is plotted in terms of different voltage of the electron beam, at frequencies of 1399, 1599 and $1799 \mathrm{MHz}$. These frequencies are third-order intermodulation frequencies (Table 2). In the range of $2200-3000 \mathrm{v}$ in all the three frequencies, the output power difference in the circuit is small.

\section{Discussion and conclusion}

As it can be seen, when voltage changes at different frequencies in TWT, the output power will change. Voltage variation is important in a particular range.

Fig. 5 Circuit output power versus axial distance in, $V=3200 \mathrm{v}$ 


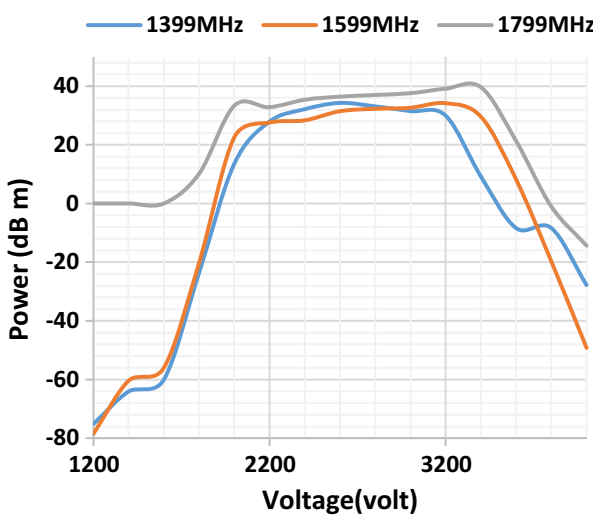

Fig. 8 Circuit output power versus beam voltage

Applying a lower voltage is needed to achieve a higher power in low frequencies, but for gaining a higher power in high frequencies, using a bigger voltage is required. In a specified voltage range, the changes in the output power are not noticeable. Beam emission voltage has a direct relationship with output power to a certain extent, but after a certain limit this relationship becomes inverse which means increasing beam emission voltage results in the decrease in output power. This happens because of the saturation phenomena which is the nonlinear operation of TWT. In smaller frequencies, saturation phenomena (this phenomena happens when increasing of the output power stops) in the TWT happen in lower voltage. For instance in Fig. 2, it can be seen that the blue plot (1400 MHz) decreases in a lower voltage (about $3200 \mathrm{v}$ ), but the orange plot $(1600 \mathrm{MHz})$ along with the gray plot $(1800 \mathrm{MHz})$ decreases in a higher frequency (about $3400 \mathrm{v}$ ) but with different slopes. Comparing Figs. 2 and 3, it is observed that at $1400 \mathrm{MHz}$ (single-tone mode), for a voltage of $2200 \mathrm{~V}$, the TWT output power is $45 \mathrm{dBm}$, while this power is $30 \mathrm{dBm}$ in the same conditions in a multi tone mode. All shapes show the fact that the voltage applied at each frequency does not create a unique power; at some voltages, the power generated at various frequencies is the same approximately.

Open Access This article is distributed under the terms of the Creative Commons Attribution 4.0 International License (http://creative commons.org/licenses/by/4.0/), which permits unrestricted use, distribution, and reproduction in any medium, provided you give appropriate credit to the original author(s) and the source, provide a link to the Creative Commons license, and indicate if changes were made.

\section{References}

1. Pierce, J.R.: Traveling Wave Tubes, pp. 3-10. D Van Nostrand, New York (1950)

2. Rowe, J.E.: Nonlinear Electron-Wave Interaction Phenomena, pp. 3-10. Academic Press Inc, New York (1965)

3. Wöhlbier, J.G., Booske, J., Dobson, I.: The multi-frequency spectral Eulerian (Muse) model of a traveling-wave tube. IEEE Trans. Plasma Sci. 30, 1063-1075 (2002)

4. Wöhlbier, J.G.: Nonlinear distortion and suppression in traveling wave tubes: insights and methods. Doctor of Philosophy's thesis, University of Wisconsin-Madison, pp. 10-22 (2003)

5. Joo, Y.D., Sinha, A.K., Park, G.S.: Simple nonlinear analysis code for helix traveling wave tube. In: Vacuum Electronics Conference, pp. 118-119 (2004)

6. Srivastava, V., Joshi, S.N.: One-dimensional nonlinear model for helix TWTs. IETE Tech. Rev. 6, 500-507 (1989)

7. Singh, A., John Scharer, E., Booske, J., Wöhlbier, J.G.: Secondand third-order signal predistortion for nonlinear distortion suppression in a TWT. IEEE Trans. Electron Devices 52, 709-717 (2005)

8. Males-iliac, N., Budimir, D., Milovanovic, B.: Improved linearization technique that uses injection of second harmonics. Microw. Opt. Technol. Lett. 38, 150-153 (2003)

9. Srivastava, V., Joshi, S.N.: Improved nonlinear model for multisignal analysis of helix TWTs. IEE Proc. 139(2), 129-134 (1992)

10. Joo, Y.-D., Sinha, A.K., Wei, Y., Park, G.-S.: Simplified approach to the nonlinear analysis in helix slow-wave-structure for a traveling wave tube. Jpn. J. Appl. Phys. 42, 7104-7111 (2003)

11. Wohlbier, J.G., Dobson, I., Booske, J.H.: On the physics of harmonic injection in a traveling wave tube. IEEE Trans. Plasma Sci. 32(3), 1073-1085 (2004)

12. Singh A.: Experimental investigation of TWT nonlinearities and distortion suppression by signal injection. A thesis for the degree of Master of Science 2003, University of Wisconsin-Madison

13. Srivastava, V., Joshi, S.N., Ravinder, B., Carter, R.G., Sinha, A.K.: Design of helix slow-wave structures for high efficiency TWTs. IEEE Trans. Electron Devices 47(12), 2438-2443 (2000)

14. Wöhlbier, J.G., Booske, J.: Mechanisms for phase distortion in a traveling wave tube. Phys. Rev. E 69, 066502 (2004)

15. Joo, Y.D., Park, G.S.: Development of a simplied one-dimensional nonlinear helix traveling wave tube code based on the lagrangian disk model. IEEE Trans. Electron Devices 48(1), 3-11 (2001)

16. Sheykhe, F., Saviz, S., Salem, M.: Comparison of the effect of annular and solid electron beams on linear and nonlinear traveling wave tube. Results Phys. 7, 4324-4331 (2017)

Publisher's Note Springer Nature remains neutral with regard to jurisdictional claims in published maps and institutional affiliations. 